\title{
Experiencia compartida de dos institutos nacionales de salud en una red de investigación en enfermería
}

\section{Experience shared two National Institutes of Health in a network of nursing research}

\author{
Sandra Sonalí Olvera Arreola, * Alicia Hernández Cantoral **
}

$\mathrm{L}$ a sociedad ha cambiado y con ella la forma de organizarnos. En un mundo globalizado donde la tecnología y los sistemas informáticos son piezas clave para la evolución, es imperante que como profesionales de enfermería establezcamos mecanismos basados en redes que potencialicen el conocimiento, la experiencia y la innovación para atender los problemas relacionados con la salud. Las redes de investigación pueden ser espacios de interacción para producir transformaciones conjuntamente sin perder identidad por parte de cada integrante; pero de manera sustancial, son espacios para validar y actualizar la producción de conocimiento mediante diferentes mecanismos. En este sentido, una red de investigación se refiere al grupo o comunidad científica de profesionales, de enfermería en este caso, donde la participación de cada uno de sus integrantes es el hilo que entreteje el entramado de la profesión; si uno de estos hilos se afloja o revienta, estaremos observando una estructura frágil y sin forma. Precisamente, éste es el objetivo primordial de una red: afianzar la enfermería como disciplina para generar nuevas alternativas científicas, tecnológicas y humanas dirigidas a proporcionar cuidados seguros $\mathrm{y}$ de calidad de una forma integral a la persona que presenta alteración en alguna de sus necesidades básicas de salud.

En este contexto, el Instituto Nacional de Cardiología (INC) y el Instituto Nacional de Pediatría (INP) han participado en investigaciones multicéntricas como integrantes de la Red de Investigación en Enfermería ENEO-UNAM, Institutos e instituciones de salud, obteniendo productos publicados en revistas de enfermería acreditadas, que han aportado resultados significativos a problemas en común de la práctica diaria. Hasta el momento la Red de Investigación en Enfermería ENEO/ UNAM mantiene estrecha colaboración con la Pontificia Universidad Javeriana de Colombia para el desarrollo de un proyecto internacional de suma relevancia para la seguridad del paciente, relacionado con los eventos adversos reportados por enfermería en unidades de cuidados intensivos.

Por otra parte, se observa claramente cómo ha ido madurando la coordinación de los encuentros académicos anuales organizados por esta Red; el eco de su convocatoria ha llegado a diferentes zonas geográficas del país, la respuesta se refleja en la participación de numerosas investigaciones, incluso se logró por el Centro de Investigación Materno Infantil Gen (CIMIGen), el premio SILANES que consistió en un estímulo económico al mejor trabajo concluido y al que está en fase de protocolo en el $6^{\circ}$ Encuentro de la Red. El trabajo en Red además de socializar los problemas comunes de las instituciones participantes y buscar soluciones a éstos, también permite el fortalecimiento del proceso de investigación, ya que la capacitación continua y el alto compromiso de cada uno de sus integrantes permiten el logro de objetivos particulares y grupales. Por ello es importante mencionar que, como parte de las estrategias de fortalecimiento y desarrollo de la investigación, la coordinación de la Red siempre ha tomado en cuenta al grupo para participar en actividades académicas impartidas por distinguidos profesores en el ámbito nacional e internacional. Asimismo, a partir del año 2012, por primera vez un integrante de la Red forma parte del Comité de Investigación de la Escuela Nacional de Enfermería y Obstetricia. Este bienio la Red estará representada por el INC.

Evidentemente, el pertenecer a una Red de Investigación en Enfermería da mayor peso al trabajo realizado en conjunto; sin embargo, no se debe perder de vista que la construcción, el desarrollo, la compartición tanto de conocimientos como de experiencias, dentro y fuera del grupo, es el objetivo final. El reto está en mantener una comunicación eficaz y una distribución equitativa de responsabilidades para facilitar un ambiente de solidaridad y afecto que fomente el trabajo en equipo.

* Maestra en Enfermería en Administración del Cuidado, Instituto Nacional de Cardiología.

** Maestra en Enfermería en Administración del Cuidado, Instituto Nacional de Pediatría. 